\title{
The Phenomenology of High-Temperature Superconductive Materials
}

\author{
K.E. Gray \\ Materials Science Division, Argonne National Laboratory, Argonne, IL 60439 \\ (email kengray@anl.gov)
}

\begin{abstract}
ict. High-temperature superconductors offer considerable promise for high current applications. Some of the it issues, such as materials, grain boundaries and dissipation, will be introduced and both the fundamental and practical aspects will be addressed. For example, the first generation powder-in-tube wires based on the bismuth cuprate have restricted high field performance at liquid nitrogen temperature due to the weak inter-bilayer coupling of magnetic vortices. The second generation coated conductor uses $\mathrm{YBa}_{2} \mathrm{Cu}_{3} \mathrm{O}_{7}$ to overcome this, but it requires a high degree of biaxial texturing in order to provide notable performance in high fields in liquid nitrogen.
\end{abstract}

\section{INTRODUCTION}

To provide an overview of superconductivity in a short space is impossible. Instead the most salient points related to the use of high-temperature superconductors (HTS) as power conductors will be briefly reviewed in order to acquaint the non-specialist with the terminology and some of the basic concepts. Those wishing to go further are directed to the general references given at the end of this article. The following sections will cover: some relevant fundamental concepts of superconductivity; limits to current carrying capacity which, in practical situations, depend on the appearance and control of magnetic vortices; and the special role that grain boundaries (GBs) play in HTS.

\section{Concepts of Superconductivity: the BCS Theory}

Superconductivity results from the condensation of the conduction electrons of a metal into Cooper pairs below a transition temperature, $\mathrm{T}_{\mathfrak{c}}$, which is material dependent. The pairing mechanism of the BCS theory (Bardeen, Cooper and Schrieffer, 1957) is by phonons. Basically, the retarded electron-ion-electron Coulomb interaction is attractive and overcomes the direct Coulomb repulsion of electrons. A simple physical picture starts with one electron passing a point and polarizing the ion lattice. The massive ions are slow to react and after the first electron leaves, the excess positive charge remains and it attracts a second electron. The resulting Cooper pairs have zero spin and zero center-of-mass momentum and a binding energy, $2 \Delta$. Note that $\Delta$ is the energy gap for excited states of unpaired electrons and that these states will be partially occupied at finite temperatures. In the high-temperature superconductors (HTS), i.e., the cuprates, the pairing mechanism may not be by phonons and the superconductive wave function exhibits a d-wave symmetry that was not considered in the BCS formulation. Nevertheless, the existence of Cooper pairs and the energy gap, $\Delta$, have been clearly demonstrated. 
Applying a transient electric field accelerates the pairs (which maintain a zero center-of-mass momentum) to a finite drift velocity, $\mathrm{v}_{\mathrm{s}}$, which defines a supercurrent state with no electrical resistance. The supercurrent density, $\mathrm{J}_{\mathrm{s}}$, is limited by pairbreaking that occurs when the kinetic energy of a Cooper pair exceeds its binding energy $2 \Delta$ : i.e., for $\mathrm{v}_{\mathrm{S}}>2 \Delta \mathrm{p}_{\mathrm{F}}$, where $\mathrm{p}_{\mathrm{F}}$ is the Fermi momentum. The depairing critical current density, $\mathrm{J}_{\text {depair }}$, from GL theory (Ginzburg and Landau, 1950) is $\sim \mathrm{H}_{\mathrm{c}} / 2 \lambda_{\mathrm{L}}$, where $\mathrm{H}_{\mathrm{c}}$ is the thermodynamic critical magnetic field and $\lambda_{\mathrm{L}}$ is the London penetration length for magnetic fields. Values of $\mathrm{J}_{\text {depair }}$ are of order $10^{7}-10^{8} \mathrm{~A} / \mathrm{cm}^{2}$ for most materials and somewhat higher for HTS. This intrinsic current limiting mechanism sets an absolute maximum for the supercurrent density but a lower value can occur in the presence of magnetic fields. Even in zero applied field, the self-field of the measuring current cannot be ignored. Thus measurements of $\mathrm{J}_{\text {depair }}$ must be done in very-small-area, isolated wires, and an example for niobium (Gabutti, et al, 1989) is shown in Fig. 1 for a film of area $\sim 0.1 \mu \mathrm{m}^{2}$. By fitting the data to the GL temperature dependence, the zero-temperature value of $\mathrm{J}_{\text {depair }}$ is within a factor of two of the above GL estimate.

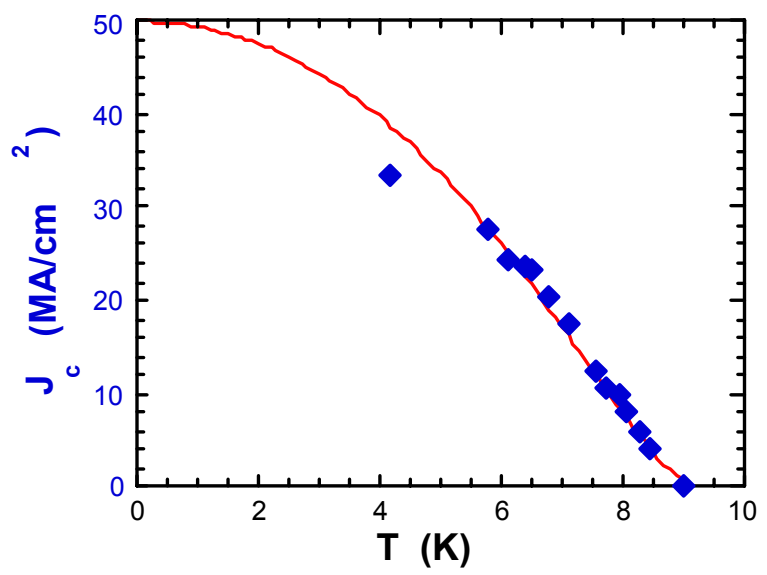

FIGURE 1. The zero-field critical current density of extremely small cross section area Nb films fit to the Ginzburg-Landau model for depairing (from Gabutti, et al, 1989). The self-field of the current in this geometry is less than the critical field of Nb.

\section{Magnetic Fields: Abrikosov Vortices}

To the first approximation, superconductors screen magnetic fields from entering their interior. This perfect diamagnetic response is the result of supercurrents that flow in a surface layer of thickness $\sim \lambda_{\mathrm{L}}$. As the field is increased beyond $\mathrm{H}_{\mathrm{c}}$ for type-I superconductors (e.g., elements like $\mathrm{Al}, \mathrm{Sn}, \mathrm{Pb}$, etc.) superconductivity is lost and the field completely fills the material. For the far more prevalent type-II superconductors (e.g., HTS cuprates and technological magnet wires using $\mathrm{NbTi}$ or $\mathrm{Nb}_{3} \mathrm{Sn}$ ), superconductivity is lost at the upper critical field, $\mathrm{H}_{\mathrm{c} 2}$, but the field enters at the lower critical field, $\mathrm{H}_{\mathrm{c} 1}$, in the form of vortex lines (Abrikosov, 1957). Circulating supercurrents, surrounding these Abrikosov vortices, result in concentrated fields of order $\mathrm{H}_{\mathrm{c}}$ at their center, which decay on a length scale of $\lambda_{\mathrm{L}}$. The total flux of each vortex, $\Phi_{\mathrm{o}}$, is quantized and equal to $2 \times 10^{-15} \mathrm{Tm}^{2}$. It is the macroscopic quantum nature of the superconducting wave function, $\psi \exp (i \phi)$, with an amplitude, $\psi$, and phase, $\phi$, which leads to flux quantization. Since currents in quantum systems are associated with a phase gradient, $\hat{\mathbf{A}} \phi$, the phase must increase continuously along the path of the circulating current. Then to maintain a single-valued wave function, the phase change once around the vortex must be an integer times $2 \pi$. It is the negative interfacial energy of type-II superconductors that stabilizes the minimum phase change of just $2 \pi$ and $\Phi=\Phi_{0}$. To satisfy the $2 \pi$-phase change, the supercurrent density diverges as $1 / \mathrm{r}$ and necessarily exceeds $J_{\text {depair }}$ at a radius the order of $\xi$, the superconducting 
coherence length. Thus the magnitude of the superconducting wave function must decrease for $\mathrm{r}<\xi$ and approach zero at the center of the vortex, where theory (Abrikosov, 1957) indicates a singularity, with $\psi(r) \sim r / \xi$.

In a defect-free superconductor, Abrikosov vortices form a regular triangular lattice. Although the regions surrounding the vortices are superconducting, the presence of vortices can affect transport supercurrents. A uniform transport current interacts with the vortices via a Magnus or Lorentz force on the vortices, that equals $\mathbf{J x} \mathbf{B}$ and is transverse to the current density $\mathbf{J}$. If the vortices are free they will move at a flux flow velocity, $\mathbf{v}_{\mathbf{f f}}$, dictated by a balance between the Lorentz force and viscous damping. Then, application of the Lorentz transformation yields an electric field, $\mathbf{E}=\mathbf{-}_{\mathbf{f f}} \mathbf{X B}$, parallel to $\mathbf{J}$ that leads to the loss of zero resistance, i.e., to dissipation.

The reason that type-II superconductors can be made useful is because vortices can be pinned by sample defects to prevent such flux flow and the ensuing dissipation. Then a balance between the Lorentz and pinning forces determines the maximum (critical) current density, $J_{\text {depin }}$, for supercurrent flow. Almost any defect will pin vortices, but non-superconducting inclusions (second-phase impurities, including radiation induced amorphous regions) that have a size scale matching the core $(\sim 2 \xi)$ are generally very effective. To see this, note that vortices residing in defect-free regions remove the superconducting condensation energy, $\mathrm{E}_{\mathrm{c}}$, over their core volume (of diameter $2 \xi$ ). However, this energy is gained back if the vortex sits on a non-superconducting inclusion so $\Delta \mathrm{E}_{\mathrm{c}}$ is in fact the pinning energy, $\mathrm{E}_{\mathrm{pin}}$, that leads to an effective pinning force $\sim \mathrm{E}_{\mathrm{pin}} / \xi$. Note that at sufficiently high temperatures, $\mathrm{k}_{\mathrm{B}} \mathrm{T}>\sim \mathrm{E}_{\mathrm{pin}}$, and then thermal activation will 'ionize' the vortices out of their pinning sites, thus leading to a finite flux flow resistance, even in the limit of zero current. Thus this condition defines the crossover from irreversible, hysteretic magnetic behavior at low temperatures to reversible behavior at high temperatures. For conventional superconductors this reversible region occurs only very close to $T_{\mathfrak{c}}$, however for HTS it can extend tens of degrees below $T_{c}$. Because the pinning energy depends on applied magnetic field, $\mathrm{H}$, this behavior is often quantified, at a given temperature, by a crossover field, $\mathrm{H}^{*}$. Above $\mathrm{H}^{*}$, vortices tend to be ionized and free and below $\mathrm{H}^{*}$ they are mostly pinned.

Due to vortex-vortex interactions (which led to the triangular lattice) and the usual random spatial distribution of pinning sites it is not simple to derive the critical current from a knowledge of the individual pinning forces, $\mathrm{E}_{\mathrm{pin}} / \xi$. This problem of the summation of pinning forces has been approached with a collective pinning model (Larkin and Ovchinnikov, 1973; see also Blatter, et al, 1994) in which discrete vortices are replaced with a continuum approximation (long wavelength limit of vortex lattice). Some success for strong pinning and short wavelengths has been recently achieved (Gray, et al., 1998a) through analyzing data for columnar pinning sites (linear amorphous tracks made by high-energy, heavy-ion irradiation, see Fig. 2a) in epitaxial films of the Tl-based HTS cuprate. In this straightforward model, the probability that a vortex is unpinned by the ion tracks, $\mathrm{P}_{\text {unpin }}$, is just given by

$$
\mathrm{P}_{\text {unpin }}=\exp \left\{-\left(\mathrm{E}_{\mathrm{pin}} / \mathrm{E}_{\mathrm{o}}\right)\left(\mathrm{B}_{\phi} / \mathrm{B}\right)\right\}
$$

where $E_{0}$ is a constant that accounts for the energy of distortion of the Abrikosov lattice and the last term is the ratio of average densities of pinning sites, $\mathrm{B}_{\phi}$, to vortices, B. By using the Bardeen and Stephen (1965) model for dissipation and including thermal activation, quite adequate fits to the low-current resistive data were found, and these are displayed in Fig. $2 b$.

\section{Vortices in the Layered HTS Cuprates}

The HTS cuprates consist of superconducting $\mathrm{CuO}_{2}$ multilayers (usually bilayers) separated by non-superconducting layers. For magnetic fields perpendicular to the layers one finds pancake vortices with their supercurrents confined 


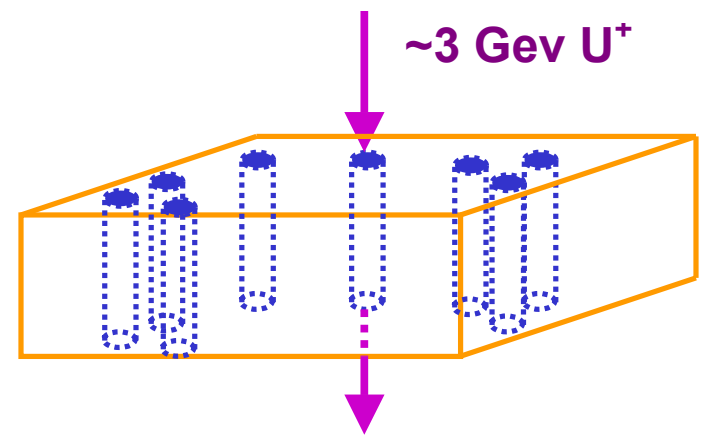

(a)

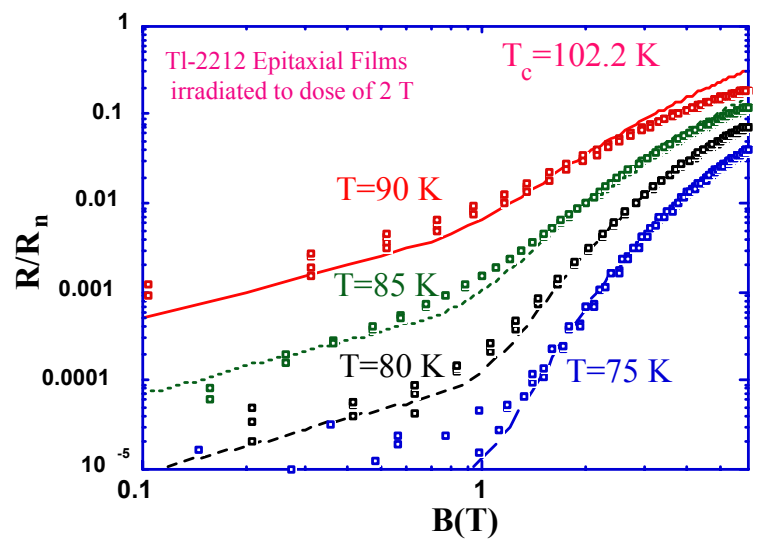

(b)

FIGURE 2. (a) The sample, a $\mathrm{Tl}_{2} \mathrm{Ba}_{2} \mathrm{CaCu}_{2} \mathrm{O}_{\mathrm{y}}$ film with $\mathrm{T}_{\mathrm{c}}=102 \mathrm{~K}$, was irradiated with $\sim 3 \mathrm{Gev}$ uranium ions to produce amorphous tracks that are $\sim 7-10 \mathrm{~nm}$ diameter at an average density equivalent to the vortex density in a field of $2 \mathrm{~T}$. (b) The resistive dissipation is shown at various temperatures with fits based on Eq. 1 and thermal activation (Gray, et al, 1998a).

to the bilayers. The establishment of well-correlated flux lines (like the Abrikosov flux lines in isotropic superconductors) requires strong coupling of pancake vortices in adjacent bilayers and that depends on the anisotropy. The coupling, or flux-line tension, is very weak in $\mathrm{Bi}_{2} \mathrm{Sr}_{2} \mathrm{CaCu}_{2} \mathrm{O}_{8+\mathrm{x}}$ (BSCCO) and fairly strong in $\mathrm{YBa}_{2} \mathrm{Cu}_{3} \mathrm{O}_{7-\delta}(\mathrm{YBCO})$. Thus flux-line-cutting processes in $\mathrm{BSCCO}$ can lead to independent flux motion in isolated bilayers, and that greatly reduces the effectiveness of pinning. Whereas in isotropic materials, a few point pinning sites can pin a long section of an Abrikosov flux line, the weak coupling in BSCCO means these sites are only fully effective within one superconducting bilayer.

Studies of the irreversibility crossover field, $\mathrm{H}^{*}$, in a number of HTS cuprates have helped to elucidate the nature of the interlayer coupling and its dependence on the spacing between $\mathrm{CuO}_{2}$ bilayers. Data from Kim, et al (1991) are shown in Fig. 3a for YBCO, BSCCO and several Tl-based cuprates. They exhibit a strong dependence on interlayer spacing and only a very weak dependence on the thickness of the superconducting layers (i.e., bilayer, trilayer, etc).

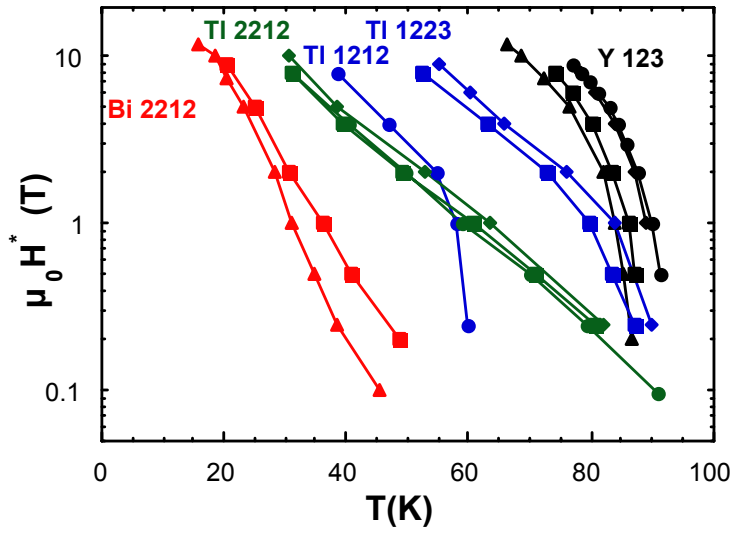

(a)

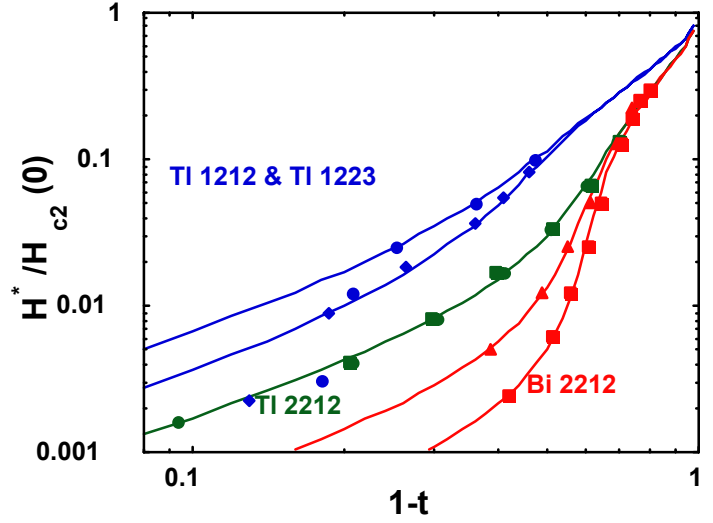

(b)

FIGURE 3. (a) The irreversibility crossover fields are shown for several samples of various HTS cuprates. (b) Fits of the data to the model described in the text (Kim, et al, 1991) demonstrate that the Josephson coupling between superconducting layers determines the effective pinning strength. 
Figure 3b shows excellent agreement with the model of Kim, et al (1991) that consists of a conventional pinning picture within superconducting layers and Josephson coupling between layers. The model successfully fits the temperature dependence of $\mathrm{H}^{*}$ with a Josephson coupling parameter that depends exponentially on the interlayer spacing, i.e., the expected dependence for Josephson tunneling. This model correctly predicted $\mathrm{H}^{*}$ for the $\mathrm{Hg}$-based cuprates from their structure before $\mathrm{H}^{*}$ was measured.

The results of Kim, et al (1991) also show that the very poor effective flux pinning of BSCCO at high temperatures and fields is an intrinsic property. The one-thallium-layer cuprates (Tl-1212 and Tl-1223) are better, but YBCO is clearly the best and thus the research emphasis for current carrying conductors has shifted to YBCO in recent years.

For fields parallel to the bilayers in BSCCO, flux enters as Josephson vortices centered between neighboring bilayers. Because of the very weak interbilayer supercurrents, the Josephson vortices, which also contain one flux quantum, $\Phi_{\mathrm{o}}$, are very much elongated in the direction of the plane of the bilayer. Nevertheless, for transport currents along the multilayers the Lorentz force, on vortices parallel to the layers, is directed perpendicular to the layers so the non-superconducting layers provide intrinsic pinning that is strong and effective. Thus the critical currents remain reasonably large even at high fields and temperatures. For intermediate angles the vortices are made up of weakly coupled chains of pancake and Josephson vortices, and dissipation rapidly approaches the poorer behavior for perpendicular fields.

\section{Grain Boundaries in HTS and Strategies to Deal with Them}

Shortly after epitaxial films of YBCO were deposited onto $\mathrm{SrTiO}_{3}$ single crystal substrates, artificial grain boundaries (GBs) were studied by depositing $\mathrm{YBCO}$ onto $\mathrm{SrTiO}_{3}$ bicrystals (Dimos, et al, 1988). The result was an exponential decrease in $\mathrm{J}_{\mathrm{c}}$ as a function of [001] tilt misorientation angle, $\Theta$. This important observation was very discouraging for current carrying applications since it implied the need for biaxially textured HTS materials. Note that in conventional superconductors, GBs are not detrimental and can even provide flux pinning to increase $\mathrm{J}_{\mathrm{c}}$. Thus early conductor research concentrated on BSCCO, which through powder-in-tube processing minimized the detrimental GB effects. However, as noted above, the poor intrinsic flux pinning of BSCCO limited its high-field potential to low temperatures $(\mathrm{T}<30 \mathrm{~K})$. Although the one-thallium-layer cuprates improved the flux pinning (Kim, et al, 1991), they did not reproduce the hoped-for GB connectivity found with BSCCO powder-in-tube processing.

A major breakthrough was the demonstration of a potentially commercially scalable process to produce biaxiallytextured YBCO deposited films. The first embodiment used ion-beam-assisted deposition (IBAD) to create biaxially textured yttria-stabilized zirconia (YSZ) films on an untextured metallic substrate (Wu, et al, 1995). Subsequently, rolling-assisted-biaxially-textured substrates (RABiTS) were made by cold-working nickel tapes (Goyal, et al, 1996). The coated conductors produced by these techniques have impressive performance, $\mathrm{J}_{\mathrm{c}} \sim 2 \times 10^{6}$

$\mathrm{A} / \mathrm{cm}^{2}$ at $77 \mathrm{~K}$. In each case, a high degree of orientation (average $\Theta<5^{\circ}$ ) is essential, and although achievable, it incurs a significant fabrication cost. Improvements in GB coupling could relax the stringent requirement on $\Theta$. It is also instructive to recognize that supercurrents will cross at least one million GBs per meter of length of coated conductor made by the IBAD process. Thus, reducing the dissipation across GBs at their operating current could lead to important improvements. Additional techniques for biaxial texture, including inclined substrate deposition (ISD), are also being studied (see Hasegawa, et al, 1997 and Bauer, et al, 1999).

A program to improve GBs must start with an understanding of the mechanisms that limit $\mathrm{J}_{\mathrm{c}}$ and result in dissipation. Grain boundaries behave like Josephson junctions that exhibit an intrinsic current carrying capacity, $\mathrm{J}_{\mathrm{cj}}$, in analogy to $\mathrm{J}_{\mathrm{depair}}$ for bulk superconductors. Like $\mathrm{J}_{\mathrm{depair}}, \mathrm{J}_{\mathrm{cj}}$ only applies directly to small-area, isolated junctions. 
Otherwise Josephson vortices appear and it is the depinning of these that determines $\mathrm{J}_{\mathrm{c}}$ in analogy with Abrikosov vortices in bulk superconductors. Here each linear GB dimension must be smaller than $\sim 4 \lambda_{\mathrm{j}}$, where $\lambda_{\mathrm{j}}$ is the length of a Josephson vortex along the junction, that is proportional to $1 / \mathrm{J}_{\mathrm{cj}}$ and is typically less than 1 micron in HTS [001] tilt GBs. Since $\lambda_{\mathrm{j}}$ is much less than typical coated conductor widths, it is necessary to consider depinning. Microscopy studies show that YBCO artificial bicrystal GBs meander on a length scale close to $\lambda_{j}$. Thus one expects strong pinning due to variations of $\mathrm{J}_{\mathrm{cj}}$ for the various facets of the meandering GB that lead to a vortex energy that is position dependent.

Theoretically, the maximum depinning current density for ideal pinning centers is of order $J_{\text {depair }}$ for Abrikosov vortices and $\mathrm{J}_{\mathrm{cj}}$ for Josephson vortices. The relative effectiveness of actual pinning sites is expressed by the fraction, $\alpha$, of that maximum, e.g., $\mathrm{J}_{\mathrm{c}}=\alpha \mathrm{J}_{\mathrm{cj}}$. Since a measure of $\alpha$ is practically impossible, it is difficult to ascertain whether it plays a role in the exponential decrease of $J_{c}$ with $\Theta$. However, the limit of negligible pinning $(\alpha=0)$ is of interest since then, dissipation occurs as soon as flux enters the GB, and the flux entry field at the edge can be directly related to the self-energy of a Josephson vortex and hence $\mathrm{J}_{\mathrm{cj}}$. In this limit, there is a specific prediction for the lowfield behavior with only one parameter, the sample dimension, w. Such low pinning may be anticipated for ideal, flat GBs made by dual-seeded, melt-textured growth (Todt, et al, 1996) and the no-free-parameter fit of the field dependence confirms this (Gray, Field and Miller, 1998b). The measured $\mathrm{J}_{\mathrm{c}}$ are shown as open symbols $\left(\mathrm{J}_{\mathrm{c} 1}\right)$ in Fig. 4 together with those of thin-film, YBCO artificial bicrystal GBs $\left(\mathrm{J}_{\mathrm{cp}}\right)$ that are shown as filled symbols.

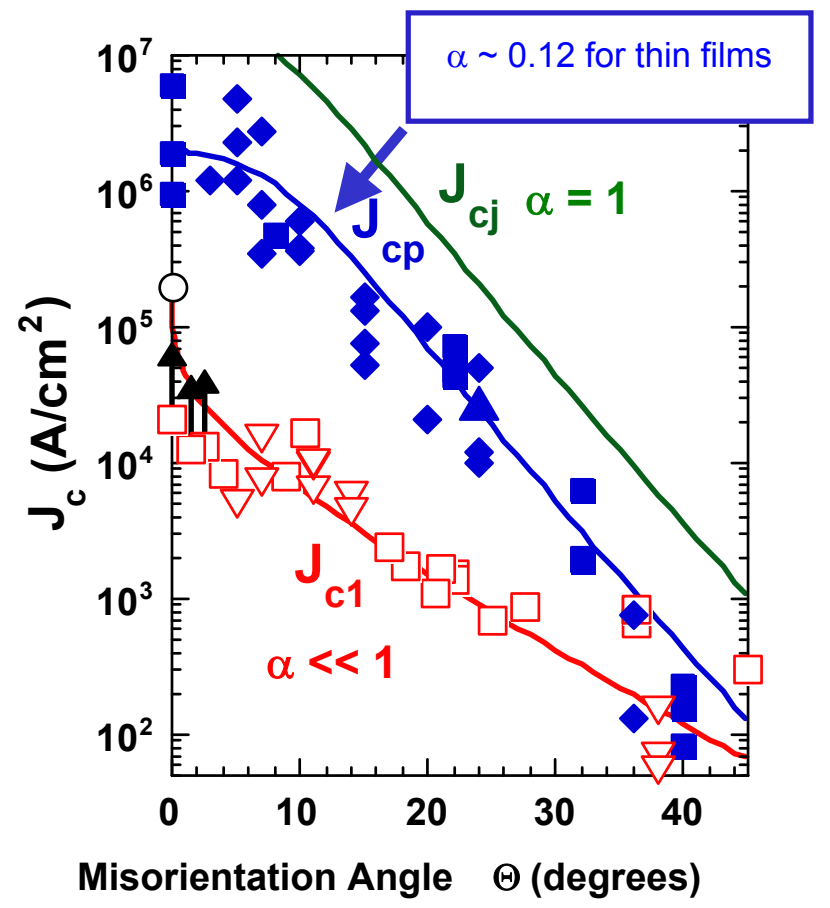

FIGURE 4. The measured critical current densities for artificial GBs in YBCO are shown as a function of the [001] tilt misorientation angle, $\Theta$. The open symbols are for bulk, flat GBs and the solid symbols for thin-film GBs. The solid lines through the data are described in Gray, Field and Miller (1998b) and the upper curve is the inferred $\mathrm{J}_{\mathrm{cj}}$ from the bulk data.

Data for the low-pinning bulk GBs then predict $\mathrm{J}_{\mathrm{cj}}$ from the expression in Gray, Field and Miller (1998b):

$$
\mathrm{J}_{\mathrm{cj}}=\pi^{2} \mathrm{w}^{2} \lambda_{\mathrm{ab}} \mathrm{J}_{\mathrm{c}}{ }^{2} / \mathrm{c} \Phi_{\mathrm{o}}
$$

where $\lambda_{\mathrm{ab}}$ is the in-plane magnetic-field penetration length for YBCO that is $\sim 150 \mathrm{~nm}$. The result of applying Eq. 2 to the bulk data of Fig. 4 is shown as the upper curve in Fig. 4 (labeled $\alpha=1$ ). Then the thin-film data show an 
average value for $\alpha$ of 0.12 , which is quite strong pinning. In the case of Abrikosov vortices the maximum experimental value of $\alpha \sim 0.25$ was obtained by Gray, et al. (1988) for the most ideal situation. It had fields parallel to a multilayer comprised of $\mathrm{NbN}$ superconducting films and AlN insulating films with optimum thicknesses that were close to the vortex core diameter, $2 \xi$. The large $\alpha$ for thin-film GBs is no doubt due to the meandering GB size scale that is also quite close to the only size scale for Josephson vortices, $\lambda_{\mathrm{j}}$, and the fact that $\mathrm{J}_{\mathrm{cj}}$ is a strong function of GB plane and not just $\Theta$. The latter fact is reinforced by the d-wave symmetry of the superconducting order parameter, $\psi$, in YBCO.

The reason for the strong $\Theta$-dependence of $J_{c}$ in GBs is not completely understood. The d-wave symmetry of $\psi$ exhibits a $\Theta$-dependence of Josephson coupling that is far too weak and the independence of $\alpha$ on $\Theta$ is inconsistent with its origin being the d-wave symmetry. The most complete theoretical description of GBs is by Gurevich and Pashitskii (1998), who can explain the strong $\Theta$-dependence by changes in the chemical potential of charge carriers (holes) that underdope the GB region. This hole depletion has its root cause in the tensile strain (lattice expansion) found in the dislocation cores that decorate GBs. Thus improved GB performance could result from: (1) additional direct doping (e.g., cation substitution) that compensates the underdoped GB; or (2) increases in the atomic density in the region of the dislocation core (e.g., by diffusing ions into these dislocation cores) that would reduce the effective tensile strain.

The seminal work to improve GB performance is the Ca doping studies of Hammerl, et al (2000) that clearly show enhanced $\mathrm{J}_{\mathrm{c}}$ in artificial YBCO thin-film GBs. The suggested mechanism was that $\mathrm{Ca}^{2+}$ ions substitute for $\mathrm{Y}^{3+}$ in YBCO and these add holes to compensate the underdoped nature of the as-made GBs. Although Ca doping of YBCO GBs yields impressive improvements in $\mathrm{J}_{\mathrm{c}}$ (up to roughly an order-of-magnitude increase), $\mathrm{Ca}$ also poisons the transition temperature, $T_{\mathfrak{c}}$, of bulk YBCO and precautions were needed to avoid this (see Hammerl, et al, 2000).

Given the above interpretation for Ca doping, it came as a surprise that $\mathrm{Sr}^{2+}$, which substitutes for $\mathrm{Ba}^{2+}$ in $\mathrm{YBCO}$, gave comparable increases in $\mathrm{J}_{\mathrm{c}}$ (Veal, et al, 2001). Similar increases to that of Ca doping were found in high-angle bulk GBs and also in a polycrystalline sintered ceramic of $\mathrm{YBCO}$, but without any noticeable degradation in $\mathrm{T}_{\mathrm{c}}$, as might be anticipated from previous studies of $\mathrm{Sr}$ substitution in YBCO. These authors suggested that the relevant mechanism is the incorporation of dopant atoms into the dislocation cores of GBs to reduce the intrinsic tensile strain that according to theory (Gurevich and Pashitskii, 1998) will diminish the detrimental hole depletion. This observation greatly expands the search for elemental dopants that might improve $\mathrm{J}_{\mathrm{c}}$ as well as provide other advantages (e.g., leaving $\mathrm{T}_{\mathrm{c}}$ unchanged or simpler processing).

\section{Summary and Further Reading}

An attempt is made to convey the most salient points related to the use of high-temperature superconductors as power conductors in order to acquaint the unfamiliar reader with the terminology and some of the basic concepts. Included are the fundamentals of the BCS theory of superconductivity and limitations to current carrying capacity which, in practical situations, depend on the appearance and control of magnetic vortices. Those wishing to go further are directed to the following general references: Tinkham (1975); Saint-James, Sarma and Thomas (1969); Orlando and Delin (1991); Barone and Paterno (1982); and de Gennes (1966). The final topic on the special role of grain boundaries in HTS describes the current state of research in a rapidly progressing area of importance for both applications and fundamental knowledge. 


\section{NOMENCLATURE}

$\alpha=$ vortex pinning force/maximum pinning force

$\mathbf{B}=$ vector magnetic induction $(\mathrm{T})$

$\mathrm{B}_{\phi}=$ equivalent density of pinning sites $(\mathrm{T})$

$\mathrm{c}=$ velocity of light $(\mathrm{m} / \mathrm{s})$

$\Delta=$ superconductive energy gap $(\mathrm{eV})$

$\mathbf{E}=$ vector electric field $(\mathrm{V} / \mathrm{m})$

$E_{c}=$ superconductive condensation energy $(J)$

$E_{0}=$ energy scale for Eq. $1(J)$

$\mathrm{E}_{\text {pin }}=$ pinning energy $(\mathrm{J})$

$\xi=$ superconductive coherence length (m)

$\Phi_{\mathrm{o}}=$ flux quantum $\left(\mathrm{Tm}^{2}\right)$

$\mathrm{H}_{\mathrm{c}}=$ thermodynamic critical magnetic field $(\mathrm{A} / \mathrm{m})$

$\mathrm{H}_{\mathrm{cl}}=$ lower critical field $(\mathrm{A} / \mathrm{m})$

$\mathrm{H}_{\mathrm{c} 2}=$ upper critical field $(\mathrm{A} / \mathrm{m})$

$\mathrm{H}^{*}=$ irreversibility crossover field $(\mathrm{A} / \mathrm{m})$

$\mathbf{J}=$ vector current density $\left(\mathrm{A} / \mathrm{m}^{2}\right)$

$\mathrm{J}_{\mathrm{c}}=$ critical current density of GB $\left(\mathrm{A} / \mathrm{m}^{2}\right)$

$\mathrm{J}_{\mathrm{cj}}=$ intrinsic critical current density of $\mathrm{GB}\left(\mathrm{A} / \mathrm{m}^{2}\right)$

$\mathrm{J}_{\mathrm{cp}}=$ critical current density of thin-film GBs $\left(\mathrm{A} / \mathrm{m}^{2}\right)$

$\mathrm{J}_{\text {depair }}=$ depairing critical current density $\left(\mathrm{A} / \mathrm{m}^{2}\right)$

$\mathrm{J}_{\text {depin }}=$ depinning critical current density $\left(\mathrm{A} / \mathrm{m}^{2}\right)$

$\mathrm{J}_{\mathrm{S}}=$ supercurrent density $\left(\mathrm{A} / \mathrm{m}^{2}\right)$

$\mathrm{k}_{\mathrm{B}}=$ Boltzman constant $(\mathrm{J} / \mathrm{K})$

$\mathrm{p}_{\mathrm{F}}=$ Fermi momentum $(\mathrm{kg}-\mathrm{m} / \mathrm{s})$

$\lambda_{\mathrm{ab}}=$ in-plane magnetic-field penetration length (m)

$\lambda_{\mathrm{j}}=$ length of Josephson vortex along the junction (m)

$\lambda_{\mathrm{L}}=$ London penetration length $(\mathrm{m})$

$\mathrm{P}_{\text {unpin }}=$ probability that a vortex is unpinned

$\phi=$ phase of superconductive wave function (radians)

$\mathrm{r}=$ radial distance from vortex core $(\mathrm{m})$

$\psi=$ amplitude of superconductive wave function

$\mathrm{T}_{\mathrm{c}}=$ superconductive transition temperature $(\mathrm{K})$

$\Theta=[001]$ tilt misorientation angle (radian)

$\mathbf{v}_{\mathbf{f f}}=$ vector flux flow velocity $(\mathrm{m} / \mathrm{s})$

$\mathrm{v}_{\mathrm{S}}=$ Cooper pair drift velocity $(\mathrm{m} / \mathrm{s})$

$\mathrm{W}=$ sample size $(\mathrm{m})$

\section{ACKNOWLEDGMENTS}

The author has benefited enormously from ongoing collaborations and discussions with the following: D.G. Steel, J.D. Hettinger, D.J. Miller, A. Gurevich, J. Mannhart, D.H. Kim, B.W. Veal, A.P. Paulikas, H. Claus, V. Todt, M.B. Field, T.J. Marks, P. Berghuis, A. Gabutti, V.M. Vinokur, A. Koshelev, M.P. Maley, S.R. Foltyn and D.K. Christen. This work is partially supported by the U.S. Department of Energy, Basic Energy Sciences-Materials Sciences- 
Metals, Ceramic and Engineering Sciences and Energy Efficiency and Renewable Energy—Superconductivity Program for Electric Systems, under contract W-31-109-ENG-38.

\section{REFERENCES}

Abrikosov, A.A., "On the Magnetic Properties of Superconductors of the Second Type", Zh.. Eksperim. i Teor. Fiz. 32, 14421452 (1957) [Sov. Phys.-JETP 5, 1174-1182 (1957)].

Bardeen, J., Cooper, L.N. and Schrieffer, J.R., "Theory of Superconductivity”, Phys. Rev. 108, 1175-1204 (1957).

Bardeen, J. and Stephen, M.J., "Theory of the Motion of Vortices in Superconductors", Phys. Rev. 140, A1197-A1207 (1965).

Barone, A. and Paterno, G., Physics and Applications of the Josephson Effect, Wiley, New York (1982).

Bauer, M., Semerad, R. and Kinder, H., "YBCO Films on Metal Substrates with Biaxially Aligned MgO Buffer Layers", IEEE Trans. Appl. Supercond. 9, 1502-1505 (1999).

Blatter, G., Feigel'man, M.V., Geshkenbein, Larkin, A.I. and Vinokur, V.M., "Vortices in High-Temperature Superconductors", Rev. Mod. Phys. 66, 1125-1388 (1994)

De Gennes, P.G., Superconductivity in Metals and Alloys, W.A. Benjamin, New York (1966).

Dimos, D., Chaudhari, P., Mannhart, J. and LeGoues, F.K., "Orientation Dependence of Grain-Boundary Critical Currents in $\mathrm{YBa}_{2} \mathrm{Cu}_{3} \mathrm{O}_{7} \delta$ Bicrystals", Phys. Rev. Lett. 61, 219-222 (1988).

Gabutti, A., Wagner, R.G., Gray, K.E. and Kampwirth, R.T., "Superconducting Detector for Minimum Ionizing Particles", Nucl. Instr. and Meth. A278, 425-430 (1989).

Ginzburg, V.L. and Landau, L.D., "On the Theory of Superconductivity", Zh.. Eksperim. i Teor. Fiz. 20, 1064-1082 (1950).

Goyal, A., Norton, D.P., Budai, J.D., Paranthaman, M., Specht, E.D., Kroeger, D.M., Christen, D.K., He, Q., Saffian, B., List, F.A., Lee, D.F., Martin, P.M., Klabunde, C.E., Hatfield, E. and Sikka, V.K., "High Critical Current Density Superconducting Tapes by Epitaxial Deposition of $\mathrm{YBa}_{2} \mathrm{Cu}_{3} \mathrm{O}_{\mathrm{x}}$ Thick Films on Biaxially Textured Metals", Appl. Phys. Lett. 69, 1795-1797 (1996).

Gray, K.E., Kampwirth, R.T., Murduck, J.M. and Capone, D.W., "Experimental Study of the Ultimate Limit of Flux Pinning and Critical Currents in Superconductors", Physica C152, 445-455 (1988).

Gray, K.E., Steel, D.G., Hettinger, J.D. and Eddy, M.M., "Modeling Flux-Flow Dissipation from Randomly Placed StrongPinning Sites and Comparison with Ion-Irradiated Cuprate Superconductors", Phys. Rev. B57, 13894-13898 (1998a).

Gray, K.E., Field, M.B. and Miller, D.J., "Explanation of Low Critical Currents in Flat, Bulk Versus Meandering, Thin-Film [001] Tilt Bicrystal Grain Boundaries in $\mathrm{YBa}_{2} \mathrm{Cu}_{3} \mathrm{O}_{7}$ ", Phys. Rev. B58, 9543-9548 (1998b).

Gurevich, A. and Pashitskii, E.A., "Current Transport Through Low-Angle Grain Boundaries in High-Temperature Superconductors”, Phys. Rev. B57, 13878-13893 (1998).

Hammerl, G., Schmehl, A., Schulz, R.R., Goetz, B., Bielefeldt, H., Schneider, C.W., Hilgenkamp, H. and Mannhart, J., "Enhanced Supercurrent Density in Polycrystalline $\mathrm{YBa}_{2} \mathrm{Cu}_{3} \mathrm{O}_{7-\delta}$ at $77 \mathrm{~K}$ from Calcium Doping of Grain Boundaries", Nature 407, 162-164 (2000).

Hasegawa, K., Yoshida, N., Fujino, K., Mukai, H., Hayashi, K., Sato, K., Honjo, S., Sato, Y., Ohkuma, T., Ishii, H., Iwata, Y. and Hara, T., "In-Plane Aligned YBCO Thin Film Tape Fabricated by Pulsed Laser Deposition", Proc. 9 $9^{\text {th }}$ Int. Symp. Supercond., Sapporo, Japan, 745-748 (1997).

Kim, D.H., Gray, K.E., Kampwirth, R.T., Smith, J.C., Richeson, D.S., Marks, T.J., Kang, J.H., Talvacchio, J. and Eddy, M., "Effect of $\mathrm{Cu}-\mathrm{O}$ Layer Spacing on the Magnetic Field Induced Resistive Broadening of High-Temperature Superconductors", Physica C177, 431-437 (1991).

Larkin, A.I. and Ovchinnikov, Yu.N., "Electrodynamics of Inhomogeneous Type II Superconductors", Zh.. Eksperim. i Teor. Fiz. 65, 1704-1714 (1973) [Sov. Phys.-JETP 38, 854-858 (1974)].

Orlando, T.P. and Delin, K.A., Foundations of Applied Superconductivity, Addison-Wesley, Reading, MA (1991).

Saint-James, D., Sarma, G. and Thomas, E., Type II Superconductivity, Pergamon, New York (1969).

Tinkham, M., Introduction to Superconductivity, McGaw-Hill, New York (1975).

Todt, V.R., Zhang, X.F., Miller, D.J., St. Louis-Weber, M. and Dravid, V.P., "Controlled Growth of Bulk Bicrystals and the Investigation of Microstructure-Property Relations of $\mathrm{YBa}_{2} \mathrm{Cu}_{3} \mathrm{O}_{x}$ Grain Boundaries”, Appl. Phys. Lett. 69, 3746-3748 (1996).

Veal, B.W., Paulikas, A.P. Claus, H., Chen, Lihua and Gray, K.E., "Strontium Doping: New Strategies for Improved Supercurrent in $\mathrm{YBa}_{2} \mathrm{Cu}_{3} \mathrm{O}_{7} \delta$ Coated Conductors and Insights into Grain-Boundary Transport Mechanisms", private communication (2001).

Wu, X.D., Foltyn, S.R., Arendt, P.N., Blumenthal, W.R., Campbell, I.H., Cotton, J.D., Coulter, J.Y., Hults, W.L., Maley, M.P., Safar, H.F. and Smith, J.L., "Properties of $\mathrm{YBa}_{2} \mathrm{Cu}_{3} \mathrm{O}_{7-\delta}$ Thick Films on Flexible Buffered Metallic Substrates", Appl. Phys. Lett. 67, 2397 (1995). 